\title{
Challenges in the management of a rare case of extensive retroperitoneal haemangioma in a pregnant woman
}

Shu-Qi $\underline{\operatorname{Tan}^{1}}$, MBBS, Jason Shau Khng $\underline{\operatorname{Lim}}^{1}$, MRCOG, MBBS, Yin Ru $\underline{\operatorname{Tan}^{2}}$, Hak Koon $\underline{\operatorname{Tan}^{1}}$, frCog, MRACOG

ABSTRACT Haemangioma of the retroperitoneal space is a rare benign capillary malformation, which can grow significantly in pregnancy due to the multiple associated cardiovascular changes. We herein describe the case of a pregnant woman with an extensive right retroperitoneal haemangioma extending from the level of the renal hilum, across the lateral anterior abdominal wall and into the thigh. We also highlight the challenges faced in the management of the patient's delivery process. To the best of our knowledge, this is the first case of such nature and severity described in the English literature.

Keywords: capillary malformation, delivery, extensive, pregnancy, retroperitoneal haemangioma

\section{INTRODUCTION}

Haemangioma of the retroperitoneal space is a rare benign capillary malformation, which can grow significantly in pregnancy due to the multiple associated cardiovascular changes. We herein report the case of a pregnant woman with an extensive right retroperitoneal haemangioma extending from the level of the renal hilum, across the lateral anterior abdominal wall and into the thigh, and describe the challenges in the management of her delivery process. To the best of our knowledge, this is the first case of such nature and severity reported in the English literature.

\section{CASE REPORT}

A 32-year-old Chinese primigravida was referred to our institution at 36 weeks of gestation for further management of her pregnancy. She had a significant history of asymptomatic extensive retroperitoneal haemangioma, involving the right lateral abdominal wall muscles, extending to the thigh. The tumour manifested as prominent blood vasculature on the skin surface, which occasionally bled upon superficial abrasion. The bleeding was easily controlled with tamponade. Routine abdominal ultrasonography done four years earlier had revealed involvement of the anterior abdominal wall. Computed tomography (CT) of the abdomen, also performed four years earlier, showed a retroperitoneal venous malformation involving the right iliopsoas, right erector spinae and right lateral abdominal wall muscles, with a superficial component in the right flank extending down to the lateral thigh. There was no involvement of the abdominal viscera. She defaulted on subsequent follow-ups, as she was asymptomatic.

The patient had been seeing a gynaecologist since Week 7 of her pregnancy, and had an uneventful antenatal course. Fetal scans were also unremarkable, with no concerns about fetal growth. However, the area of superficial blood vasculature appeared to have grown wider (Fig. 1a) over time, and towards the later gestation period, the right-sided haemangioma deviated from the womb to the left side of the abdomen. In view of the potential complexities involved, the patient was referred to our institution for the management of her delivery.

At 37 weeks of gestation, magnetic resonance (MR) imaging was performed to evaluate the location and extent of the haemangioma (Figs. 1b-d). The images showed an increase in size and spread of the haemangioma, in comparison to the earlier CT images. The tumour extended from the level of the renal hilum to the hemipelvis, inguinal region, flank and anterior thigh, spanning at least $38 \mathrm{~cm}$ in length. Within the right retroperitoneal space, the venous structures infiltrated the right psoas and iliacus muscles and displaced the right external iliac vessels medially. The uterus was consequently displaced to the left. The haemangioma extended along the right anterior abdominal wall musculature, with significant infiltration of the anterior hip and thigh muscles. Possible infiltration of the liver and spleen was also noted.

Careful vaginal examination suggested that the genital tract was not involved. The mode of delivery was comprehensively discussed with the couple in view of the extensive haemangioma, the possibility of high output cardiac failure from shunting of large volumes of blood, and the potentially life-threatening haemorrhage due to platelet consumption from the lesion (i.e. Kasabach-Merritt syndrome). Input from a multidisciplinary team, involving maternal-fetal specialists, radiologists, interventional radiologists, anaesthesiologists, haematologists, vascular surgeons and plastic and reconstruction surgeons, was collectively used to guide clinical management. Clinical examination at 38 weeks of gestation showed that the fetus was in oblique lie, with the fetal head was at the left iliac fossa. In view of the unstable lie, the couple agreed on a planned Caesarean section under controlled circumstances.

An uncomplicated Caesarean delivery of the baby was performed at Week 39 of gestation via a left paramedian incision, in view of the involvement of the right anterior abdominal wall.

${ }^{1}$ Department of Obstetrics and Gynaecology, Singapore General Hospital, ${ }^{2}$ Yong Loo Lin School of Medicine, National University of Singapore, Singapore

Correspondence: Dr Shu-Qi Tan, Final Year Resident, Department of Obstetrics and Gynaecology, Singapore General Hospital, Outram Road, Singapore 169608. rainz_bow@yahoo.com.sg 

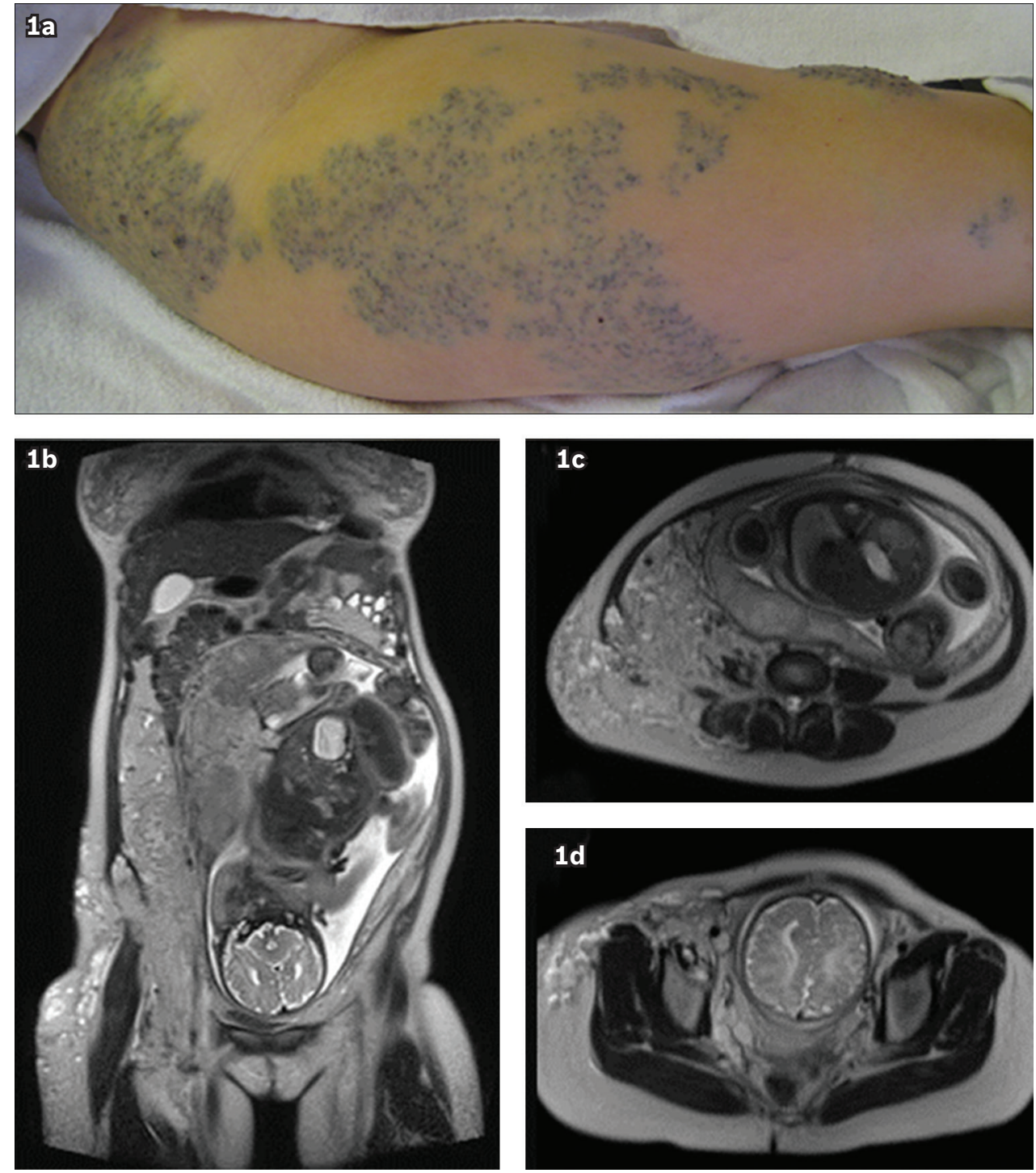

Fig. 1 (a) Photograph shows the superficial appearance of the haemangioma. (b) Coronal MR image shows the extent of retroperitoneal haemangioma. Sagittal MR images show (c) involvement of the anterolateral abdominal wall; and (d) the position of the haemangioma in relation to the uterus.

Intraoperatively, extensive haemangioma was noted at the right abdominal wall, with extension into the anterior left abdominal wall. Much care was taken to avoid potential intra-abdominal areas of involvement by the haemangioma. There was no excessive haemorrhage, and the patient had an uneventful recovery.

\section{DISCUSSION}

The current literature suggests that most haemangiomas resolve spontaneously by age nine.(1) We describe an unusual case of persistent haemangioma of the retroperitoneal space with expansion during pregnancy. This is likely due to the vasoactive factors secreted in pregnancy, resulting in peripheral vasodilation.

Caesarean section was the choice of delivery method in our case, as the fetus was in oblique lie; however, this raises concerns with regard to possible haematological complications. As there was evidence of bleeding into the haemangioma at the superficial areas (Fig. 1a) when the patient wore tight clothing (e.g. pants), massive rupture of the haemangioma during intraoperative handling was a major concern, in view of the extensive nature of the haemangioma spanning almost the entire right half of the anterior abdominal wall. Furthermore, the shunting of large volumes of blood within this retroperitoneal lesion, coupled with the huge increase in cardiac output during pregnancy, places enormous stress on the heart, which puts the patient at risk of high-output cardiac failure. Any haemorrhage within this lesion could lead to massive consumption of platelets and clotting factors within this reservoir of capillaries, and if not controlled, can result in potentially life-threatening haemorrhage(2) and disseminated intravascular coagulation. Moreover, there is a hypothetical tendency for thrombosis within such haemangioma, ${ }^{(3)}$ which is compounded by recent surgery.

The caveat to managing such pregnancies lies in comprehensive radiological evaluation, multidisciplinary orchestration, and detailed discussion with the patient. In our case, the diagnostic radiologist had preoperatively mapped out the extent of the haemangioma, particularly the retroperitoneal 
areas with the associated anastomosis. The interventional radiologist collaborated to decide on the feasibility of prophylactic insertion of arterial/venous occlusion stents to prevent massive haemorrhage. However, no stent was eventually inserted due to the extensive nature of the haemangioma and the technical difficulty in identifying the primary origin of the tumour. The hepatobiliary surgeons were activated in view of the infiltration of the haemangioma into the liver and spleen, as noted from the preoperative MR images. Good anaesthesia support is also essential. Together with the anaesthesiologist, it was decided that Caesarean section would be performed under general anaesthesia due to the potential risk of massive haemorrhage, which would require further surgery such as hysterectomy and ligation of the vascular anastomoses. The hospital's blood bank was also notified, should blood products be needed in the event of massive haemorrhage.

In addition, preoperative optimisation of the patient's cardiovascular status is essential due to the potential risk of high-output cardiac failure in the event of massive haemorrhage. Placement of arterial and venous catheters for resuscitative and monitoring purposes was also discussed with the patient and her relatives. This discussion included assessment for the need for postoperative monitoring in the intensive care unit. Detailed and extensive counselling was paramount in her management.

Elective Caesarean section was planned at 39 weeks of gestation, before the patient went into spontaneous labour, so as to conduct the delivery in a controlled fashion, with multidisciplinary support available. A left paramedian incision and a transverse lower uterine segment incision (slightly deviated to the left) were used to avoid the anterior abdominal haemangioma, which was located predominantly on the right. Due care was taken during gentle retraction by the assistants so as to minimise contact with and unnecessary tears into the haemangioma and its anastomoses during surgical exposure. The Caesarean section proceeded smoothly without any major complications or haemorrhage. Active participation of the surgeons and intervention radiologists was not needed. Intensive surveillance of the patient's cardiovascular status was crucial during the postoperative period to ensure there was no disruption to her haemodynamic condition due to the haemangioma.

Postnatally, the patient was well. Although there was no follow-up MR imaging for the haemangioma in view of cost issues, there was clinical evidence of shrinkage of the superficial part of the haemangioma. Family planning was discussed in view of the potential recurrence of the aforementioned problems in the next pregnancy. The role of therapeutic vascular embolisation, versus radiotherapy, remains a potential option to be explored in such cases, so as to optimise pregnancy outcomes. ${ }^{(4)}$ Future research in such modalities of treatment is important for holistic management in this group of patients.

\section{ACKNOWLEDGEMENTS}

We would like to thank the patient, to whom this report relates, for giving her written consent for the publication of the case, including the use of photographic illustration.

\section{REFERENCES}

1. Metry DW. Epidemiology, pathogenesis, clinical features, and complications of infantile haemangioma. In: UpToDate [online]. Available at: http://www.uptodate.com/contents/epidemiology-pathogenesisclinical-features-and-complications-of-infantile-hemangiomas. Accessed March 1, 2012.

2. Kumar P, Clark ML. Clinical Medicine. 5th ed. United Kingdom: Saunders, 2002: 1371-2.

3. Powis SJA, Rushton DI. A case of retroperitoneal haemangioma. Br J Surg 1972; 59:74-6.

4. Ogura $Y$, Watanabe K, Hosogane N, et al. Severe progressive scoliosis due to subcutaneous cavernous haemangioma: A case report. Scoliosis 2011; 6:3. 